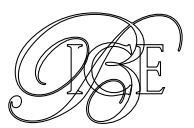

AMENET*

$M F W 4 A^{*}$

\section{EL SECTOR FINANCIERO EN ÁFRICA}

Este artículo es el primero de una serie que es resultado de la colaboración entre la red universitaria AMENET y la iniciativa Making Finance Work for Africa (MFW4A). A modo de introducción, el artículo se limita a la descripción general de los avances más notables que se han producido en el desarrollo del sector financiero en África desde los años noventa. La transformación se ha acelerado tras la crisis financiera de 2008 con la sustitución progresiva de los bancos europeos y americanos que dominaban el sector por un grupo dinámico de entidades panafricanas, principalmente de Sudáfrica, Nigeria y Marruecos. Esta misma dinámica se ha trasladado al sector de seguros y de los mercados financieros. Las bolsas han pasado de ser nueve en 1989 a treinta en 2020 y se encuentran en un proceso de interconexión muy avanzado que ha ampliado la liquidez y la diversificación de productos. Otros dos aspectos destacados del proceso han sido el avance de la inclusión financiera gracias a la penetración de la banca telefónica, en la que África es líder mundial, y el refuerzo de la regulación y los sistemas de supervisión. Esta transformación financiera está favoreciendo el proceso de integración económica a nivel continental y la interconexión con los mercados financieros a escala mundial.

Palabras clave: mercados financieros en general, instituciones y servicios financieros.

Clasificación JEL: G10, G20.

\section{Introducción}

El sector financiero en África ha experimentado un notable desarrollo desde la primera

*Este artículo es el resultado de la colaboración entre la red AMENET, proyecto impulsado por la Universidad Autónoma de Madrid (UAM) y la iniciativa Making Finance Work for Africa (MFW4A). El equipo estaba integrado por los investigadores de MFW4A dirigidos por Abdelkader Benbrahim, coordinador de partenariados (Hugues Kamewe Tsafack, Guy Menan, Muazu Ibrahimd y Alain Stephane Moulote), y los doctorandos de la red AMENET, coordinados por el profesor de la UAM Fernando Gallardo (Albert A. Agyemang-Badu y Victoria Opoku).

Los autores expresan su reconocimiento a Juan Antonio Obregón, anterior asesor principal en el Consejo del Banco Africano de Desarrollo (BAfD), por su apoyo a esta iniciativa de colaboración entre AMENET y MFW4A.

Versión de mayo de 2021.

DOI: https:/doi.org/10.32796/bice.2021.3135.7200 oleada de reformas en los años noventa que eliminaron los principales corsés de la actividad crediticia y la formación de precios, y privatizaron una buena parte de las entidades financieras estatales. Con la eliminación de buena parte de las barreras de entrada y salida y la mejora de la supervisión tras la grave crisis del sector en la década de 1980, los sistemas financieros en todo el continente han experimentado una transformación profunda.

Tradicionalmente, la investigación sobre el sector financiero africano ha prestado mucha atención a la comparación con otros mercados más desarrollados poniendo demasiado $D$ 


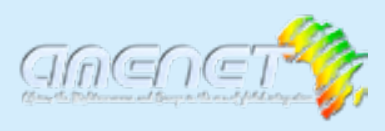

El proyecto AMENET (Africa, Mediterranean and Europe Network), liderado por la Universidad Autónoma de Madrid, es una de las redes universitarias financiadas por el programa Jean Monnet Networks de Unión Europea. AMENET reúne a un equipo multidisciplinar de investigadores de una red universitaria formada por catorce universidades africanas y europeas. El principal objetivo es promover la colaboración entre investigadores, profesionales y funcionarios africanos y europeos con el fin de profundizar en la integración económica de África con Europa y avanzar en el desarrollo económico y social sostenible en el continente africano. La red promueve la investigación de estudiantes y profesores sobre todos los temas relacionados con estos objetivos y la colaboración con diversas instituciones con intereses afines. Entre las actividades llevadas a cabo por AMENET destaca la celebración de congresos internacionales (Tánger, noviembre de 2019; Las Palmas, octubre de 2020; Accra, febrero de 2021; y Dakar, septiembre de 2021) y los intercambios bidireccionales de estudiantes y profesores africanos y europeos. En este marco se han desarrollado ya varias tesis doctorales.

\section{Making Finance Work for Africa}

Making Finance Work for Africa (MFW4A) es una iniciativa establecida por el G8 en 2007 con la colaboración de varios donantes e instituciones, y dedicada a promover el desarrollo del sector financiero en África. Gracias a la experiencia acumulada por sus promotores y socios, MFW4A reúne a los líderes de la industria, Gobiernos, instituciones de desarrollo y el mundo académico para la promoción del conocimiento aplicado al desarrollo del sector financiero en África. La iniciativa está basada en el convencimiento de que el sector financiero es un motor clave de la inversión privada, la generación de empleo y el crecimiento económico. Actualmente, el trabajo de MFW4A cubre cuatro pilares principales: 1) inclusión e innovación financiera; 2) sistemas bancarios; 3) financiación comercial; y 4) financiación a largo plazo. La secretaría de MFW4A se estableció en el año 2008 en la sede del Banco Africano de Desarrollo (BAfD), en Abiyán.

énfasis en el tamaño relativamente pequeño de las entidades bancarias, los problemas de capitalización y las debilidades de la supervisión bancaria. La mayoría de los análisis insisten, también, en el tamaño reducido de los mercados financieros, la escasa liquidez y la falta de infraestructuras financieras desarrolladas.

Sin embargo, con cierta retrospectiva, este análisis pasa por alto muchos aspectos clave del progreso realizado desde los años noventa que apuntalan el potencial de crecimiento del sector.
Las tecnologías de la información han tenido un impacto positivo en el acceso a la financiación y la diversificación y expansión de los servicios financieros. De acuerdo con los datos de la base Global Findex, la proporción de hogares con una cuenta aumentó del $23 \%$ en 2011 al $43 \%$ en $2017^{1}$. La industria de $\triangleright$

\footnotetext{
La base de datos Global Findex (https://globalfindex.worldbank. org/), financiada por la Fundación Bill y Melinda Gates, actualiza cada tres años desde 2011 la información más completa disponible sobre el progreso de la inclusión financiera en el mundo.
} 
seguros también ha atraído la atención de los principales actores globales por el potencial para el crecimiento y la diversificación de productos en el mercado.

Esta perspectiva del sector financiero presta más atención a los avances concretos que al análisis comparativo y tiene en cuenta también, lógicamente, el contexto socioeconómico en el que operan las instituciones y mercados. África representa el $16,3 \%$ de la población mundial, pero solo el 2,9\% del producto interno bruto mundial (PIB mundial) y sigue dominada por su dependencia del sector agrícola y la exportación de materias primas. Esta situación está cambiando gradualmente. El crecimiento económico en África ha tenido una tendencia ascendente y la tasa de crecimiento del PIB real ha sido del 4,9\% anual en promedio entre 2000-2008, lo que duplica el ritmo de los años ochenta y noventa (Banco Africano de Desarrollo, 2020). Se estima que, en 2050 , el $50 \%$ de la población mundial menor de veinticinco años estará en África, que previsiblemente será el continente más poblado, con 1.200 millones de personas (ONU/DESA, 2017). Aunque el fenómeno de la inmigración ha recibido una atención desproporcionada, el porcentaje de africanos desplazados fuera de su país es inferior al 2,5\% de la población actual y los movimientos migratorios se concentran principalmente dentro de África (The Economist, 26 de marzo de 2020). Todas las estimaciones apuntan a que África será el continente más poblado y uno de los principales mercados en el mundo.

El desarrollo del sector financiero se ha convertido en un elemento central del debate político en África debido a su importancia para estimular el crecimiento económico inclusivo necesario para alcanzar los Objetivos de Desarrollo Sostenible (ODS).
El objetivo de este artículo es presentar una descripción general de esta evolución relativamente reciente en el sector financiero africano. Sin abarcar de forma exhaustiva todos los temas, se han seleccionado aquellos aspectos más destacados e ilustrativos de la transformación que está en marcha.

Tras una breve descripción de la evolución general, se agrupan el resto de asuntos en cuatro secciones: 1) sector bancario; 2) inclusión financiera, que analiza el impacto de las nuevas tecnologías; 3) la financiación a largo plazo, que incluye la evolución de los mercados de capital y de deuda y las referencias a los principales inversores institucionales y públicos; y 4) un breve repaso a los principales aspectos de la regulación y la supervisión financieras.

Esta introducción se completará con otros dos artículos, el primero dedicado a la evolución reciente de las instituciones financieras y el segundo con una descripción más detallada de los mercados y la innovación financiera.

Este trabajo marca también el inicio de la colaboración entre la red universitaria Jean Monnet, AMENET y la plataforma MFW4A, que tiene como objetivo fomentar los vínculos entre jóvenes investigadores académicos en Europa y en África y los profesionales del sector financiero en el continente.

\section{Evolución del sistema financiero de África}

Desde la década de 1980, África se ha enfrentado a una gran cantidad de desafíos que han afectado al desarrollo de su sector financiero. La inestabilidad macroeconómica y sociopolítica a finales de la década de 1970 y principios de la de 1980 desencadenó una $\triangleright$ 
crisis bancaria sin precedentes que agravó la estabilidad financiera y los desequilibrios fiscales. Las dificultades financieras fueron especialmente graves para los bancos comerciales y las entidades financieras estatales. La solución de la crisis financiera implicó un coste muy elevado que lastró el desarrollo del sector financiero durante los noventa.

Sin embargo, la crisis dio también un impulso muy fuerte a reformas políticas y económicas de gran calado. En la mayoría de los países africanos, los programas de ajuste estructural promovidos por el Fondo Monetario Internacional (FMI) y el Banco Mundial incluyeron reformas generalizadas del sector financiero como un compromiso político decidido que se mantiene hasta la actualidad (Cuadro 1).

Estas políticas han conseguido minimizar el número y la gravedad de las crisis bancarias y han favorecido el desarrollo de sistemas financieros más diversificados con instituciones de captación de depósitos más eficientes, una industria de seguros más sofisticada y mercados financieros más dinámicos. Dos claves importantes de esta nueva orientación han sido el reconocimiento de la importancia de las instituciones de microfinanzas (IMF) y del desarrollo del marco regulatorio y la supervisión.

Históricamente, las microfinanzas en África han evolucionado en dos etapas diferentes. Durante el decenio de 1970 los intermediarios financieros dominantes eran las cooperativas, cajas de ahorro rurales y postales, especialmente en África Oriental y Occidental. En las décadas de 1980 y 1990 se registró un crecimiento de las organizaciones no gubernamentales de crédito respaldadas por instituciones internacionales de desarrollo y organizaciones privadas que potenciaron el desarrollo del sector financiero no bancario desde el final de la década de 1990. Gradualmente, la mayoría de los países africanos han incluido las IMF en su regulación financiera y su desarrollo ha mejorado el acceso a la financiación y ha aumentado la competencia por los depósitos con los bancos comerciales ${ }^{2}$.

La evolución del sector financiero durante los dos últimos decenios también se ha caracterizado por la proliferación de bancos extranjeros tras las privatizaciones de principios de los noventa, así como por el desarrollo de instituciones financieras panafricanas (bancos y compañías de seguros). El número de bancos panafricanos (PAB, por sus siglas en inglés) se triplicó entre 2002 y 2017 . El rápido aumento de los PAB fue impulsado principalmente por la reducción de los grupos bancarios tradicionales europeos y estadounidenses después de la crisis financiera mundial de 2008. Este aumento trajo una mayor competencia en la intermediación financiera y, como resultado, estimuló significativamente los mercados interbancarios de África y se ha convertido en la principal fuerza impulsora de la integración regional.

Los avances en términos de estabilidad han sido también notables. La mayoría de los sistemas bancarios africanos demostraron gran resistencia a la crisis financiera mundial de 2008 , como resultado de una menor exposición al riesgo derivado de las hipotecas subprime y la mejora de la calidad de la regulación y la supervisión bancaria de las dos últimas décadas. Los Gobiernos africanos están firmemente comprometidos a alinear la regulación y supervisión financieras con las mejores prácticas internacionales, mejorando gradualmente la capitalización y la liquidez del sistema.

\footnotetext{
2 Entre los perfiles financieros que MFW4A está desarrollando de la mayoría de los países africanos ilustra bien esta tendencia el caso de Senegal, en el que se han registrado tan solo 28 entidades de crédito y 19 compañías de seguro, pero hay 208 instituciones de microfinanzas que lideran la tasa de penetración de los servicios de microfinanzas en África del Oeste, y llegan al 18,4\% de la población del país (https://www.mfw4a. org/country/senegal).
} 
CUADRO 1

PRINCIPALES REFORMASY OBJETIVOS

\begin{tabular}{|l|l|}
\hline \multicolumn{1}{|c|}{ Reformas } & \multicolumn{1}{c|}{ Objetivos } \\
\hline Liberalización del crédito y tipos de interés preferenciales & $\begin{array}{l}\text { Intermediación financiera efectiva enfocada hacia sectores } \\
\text { productivos }\end{array}$ \\
\hline $\begin{array}{l}\text { Reestructuración y privatización de bancos estatales y otras } \\
\text { instituciones financieras }\end{array}$ & $\begin{array}{l}\text { Eliminar las interferencias políticas y mejorar la eficiencia del sector } \\
\text { bancario }\end{array}$ \\
\hline $\begin{array}{l}\text { Introducción de regulaciones microprudenciales y refuerzo } \\
\text { de la supervisión del Banco Central }\end{array}$ & $\begin{array}{l}\text { Asegurar la estabilidad y la rentabilidad de las instituciones } \\
\text { financieras } \\
\text { Mejorar la solvencia y reducir la tasa de morosidad }\end{array}$ \\
\hline Fuente: elaboración propia. & \multicolumn{2}{|l}{} \\
\hline
\end{tabular}

El desarrollo del sector financiero también ha traído riesgos adicionales derivados de la diversificación y la innovación de productos y servicios, la complejidad creciente de los mercados financieros, una integración regional más profunda y la interconexión más intensa con los mercados financieros globales.

Queda aún un largo camino por recorrer. La financiación en África sigue siendo insuficiente en escala, demasiado cara y muy orientada hacia el corto plazo. Los sistemas bancarios son reducidos y demasiado concentrados, con una capacidad limitada de movilización del ahorro local para financiar actividades productivas en el sector real. Las imperfecciones del mercado, como la asimetría de la información y la ineficiencia en la asignación de recursos, inducen un coste elevado del endeudamiento que actúa como principal freno a la inclusión financiera. La profundidad y la liquidez de los mercados financieros avanzan aún muy lentamente para asegurar que la intermediación financiera tenga el impacto necesario para alcanzar los objetivos de financiación marcados por la Agenda 2030.

A pesar de todas estas limitaciones, la creciente diversidad de proveedores de servicios ofrece una gran oportunidad para acelerar la innovación e integrar a una gran mayoría de la población que sigue sin tener acceso. Estas nuevas tendencias, sin duda apoyadas en las tecnologías digitales y los nuevos modelos de negocio, como evidencia el rápido desarrollo de la banca telefónica en todo el continente, ofrecen un atisbo de esperanza de que el sector financiero en África consolide su potencial como motor de un crecimiento económico más inclusivo y sostenible.

\section{El sector bancario}

Muchos países africanos han experimentado un crecimiento significativo y una expansión y profundización de sus sistemas bancarios. Hoy en día, el continente es el segundo mercado bancario de más rápido crecimiento en el mundo, considerando tanto la banca minorista como la mayorista. Los bancos comerciales continúan dominando el sector bancario en África, a excepción de Sudáfrica, y, por tanto, la intermediación financiera en todo el continente. La profundidad y cobertura de los sistemas financieros, medida por las relaciones del crédito al sector privado y el dinero en sentido amplio (M2) con el PIB, ha aumentado en las últimas décadas, aunque sigue siendo menor en comparación con otras economías emergentes.

La liberalización financiera y las reformas que siguieron, las mejoras en la capacidad institucional y reguladora y la expansión de las actividades transfronterizas, gracias al $\triangleright$ 
CUADRO 2

EXPANSIÓN DE LOS SERVICIOS FINANCIEROS EN ÁFRICA (2015-2019)

\begin{tabular}{|c|c|c|c|c|c|}
\hline & 2015 & 2016 & 2017 & 2018 & 2019 \\
\hline 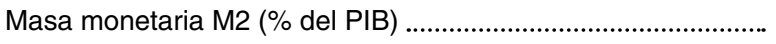 & 42,44 & 44,14 & 43,72 & 43,06 & 46,23 \\
\hline Crédito al sector privado ( $\%$ del PIB) & 27,22 & 27,73 & 27,17 & 24,94 & 27,55 \\
\hline Crédito bancario al sector privado (\% del PIB) .............................. & 24,39 & 24,83 & 24,12 & 23,35 & 24,93 \\
\hline
\end{tabular}

rápido desarrollo de las entidades bancarias panafricanas, han transformado significativamente el panorama del sector bancario ${ }^{3}$.

Los sectores bancarios en África son ahora relativamente más profundos y estables y las crisis bancarias sistémicas han disminuido drásticamente en las últimas dos décadas. El Cuadro 2 confirma que el desarrollo financiero, medido por el crédito interno al sector privado, sigue siendo insuficiente, aunque los niveles de capitalización se encuentran por encima del requisito del $10,5 \%$ fijado en los Acuerdos de Basilea, lo que ilustra bien una mayor capacidad de los bancos para resistir las perturbaciones (Gráfico 1).

Además de los niveles de adecuación del capital, la rentabilidad de los bancos, tanto por el rendimiento de los activos como por el capital, también continúa creciendo. Según Chironga et al. (2018), el sector bancario de África es el segundo más rentable (detrás de América Latina y comparable a Asia emergente y Oriente Medio), con un rendimiento sobre el capital de más del doble del promedio mundial del $9 \%$.

El coste de la financiación es aún elevado a pesar del aumento de la competencia en

3 El Banco Europeo de Inversiones (BEI) elabora periódicamente un informe sobre la banca en África (Banking in Africa). En 2015, la segunda edición incluyó los resultados de una encuesta sobre la expansión de los bancos panafricanos. MFW4A ha colaborado con el BEI en esta tarea. Más recientemente, el programa DEGRP (Development and Economic Growth Research Programme) de ODI (Overseas Development Institute) también ha analizado la expansión de las subsidiarias de bancos con sede en países africanos, principalmente de Sudáfrica, Nigeria y Marruecos (Raga \& Tyson, 2021a). En este proceso, destaca la expansión de tres bancos marroquíes (AWB, BCPE y BCME), que ha sido analizada con detalle en un informe publicado en 2019 por el Ministerio de Economía y Hacienda de Marruecos (Boumahdi, 2019). los sistemas bancarios. Esto se debe a que los bancos mantienen el exceso de liquidez y de deuda pública, principalmente en bonos y letras a corto plazo. Debido a la alta rentabilidad y el menor riesgo de la deuda pública, esta inversión se realiza a expensas del crédito al sector privado necesario para promover el crecimiento económico crowding-out ${ }^{4}$.

También ha habido mejoras en los últimos años con respecto a la adopción de recomendaciones internacionales sobre límites prudenciales y estabilidad del sector financiero. El último informe del Banco Europeo de Inversiones (BEl, 2020) confirma que la mayoría de los grupos bancarios cumplen con los Acuerdos I y II de Basilea, y una buena parte ya cumplen o trabajan para aplicar los de Basilea III.

\section{Inclusión financiera}

El acceso a la financiación ha mejorado notablemente, aunque persisten las disparidades. Según Global Findex, el porcentaje de adultos en África Subsahariana con acceso a una cuenta ha aumentado de 34 en 2014 a 43 en 2017, todavía por debajo del promedio mundial del $67 \%$. La difusión de los servicios de dinero móvil es el instrumento $\square$

\footnotetext{
4 La mayoría de los mercados interbancarios del África Subsahariana surgieron en la década de 1990, pero aún se encuentran en las primeras etapas de desarrollo. En Kenia, Malawi, Uganda y Zambia, la actividad interbancaria ya representa alrededor del $30 \%$ del PIB (Raga y Tyson, 2021b).
} 


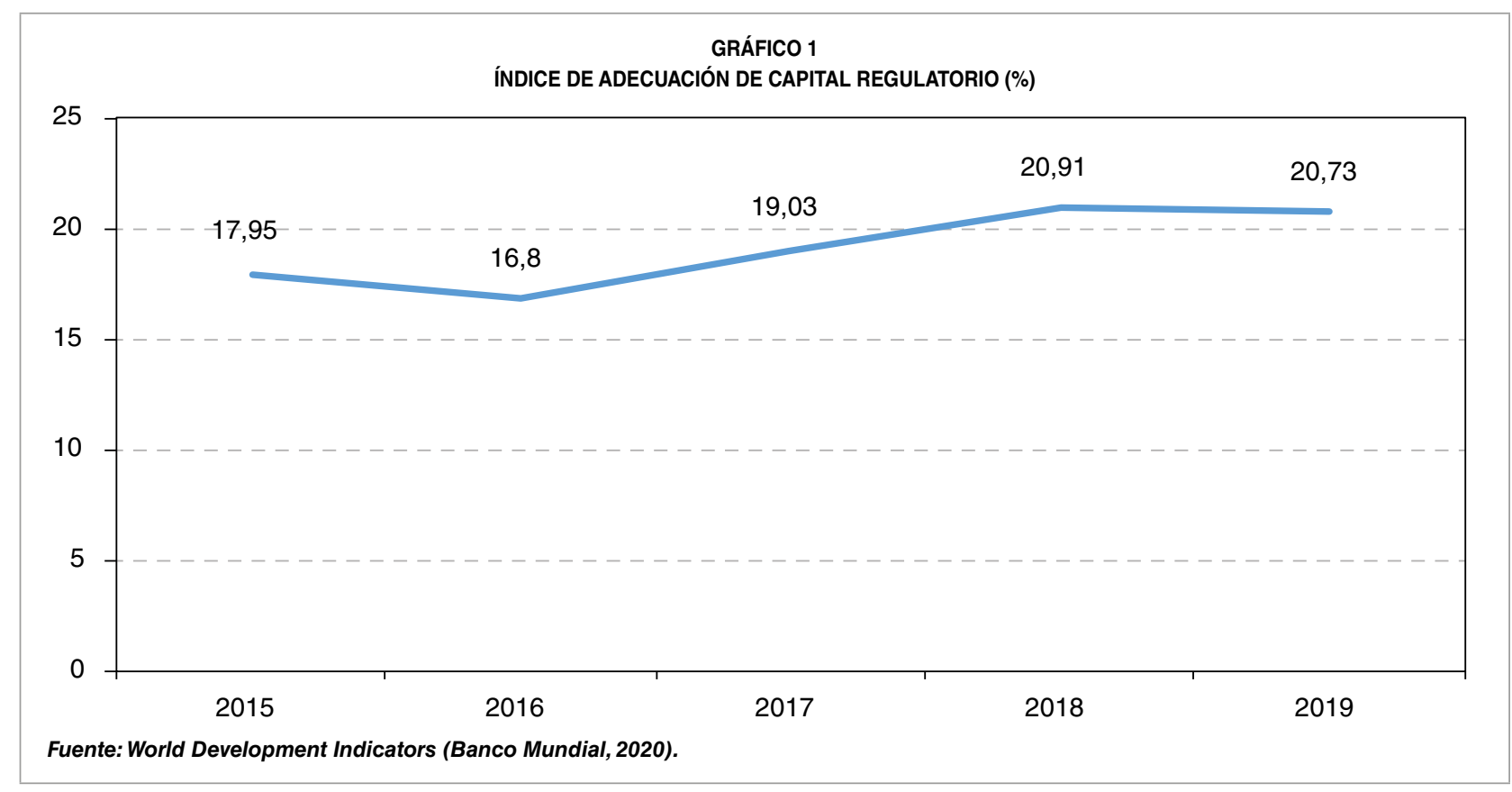

CUADRO 3

RENTABILIDAD BANCARIA EN ÁFRICA (2010-2017)

\begin{tabular}{|c|c|c|c|c|c|c|c|c|}
\hline Rentabilidad & 2010 & 2011 & 2012 & 2013 & 2014 & 2015 & 2016 & 2017 \\
\hline Rendimiento de activos (\%, antes de impuestos) ............ & 25,37 & 25,83 & 25,06 & 24,89 & 23,91 & 24,97 & 25,57 & 27,27 \\
\hline Rendimiento de capital (\%, antes de impuestos). & 25,46 & 25,95 & 25,16 & 24,93 & 23,93 & 25,03 & 25,63 & 27,38 \\
\hline
\end{tabular}

que más ha contribuido a impulsar la apertura de cuentas en muchos países, superando rápidamente las cuentas tradicionales. El porcentaje de cuentas de dinero móvil en África Subsahariana casi se duplicó entre los años 2011 y 2017, de 12 a 21, lo que confirma el peso de la tecnología y la innovación en el aumento de la inclusión financiera en el continente.

\subsection{El papel de los bancos y las instituciones microfinancieras}

El Cuadro 4 reúne varios indicadores de la situación de la inclusión financiera. Aproximadamente el $60 \%$ de la población de África todavía no tiene acceso a una cuenta bancaria formal con una institución financiera o proveedor de dinero móvil. Otra medida de la penetración de los servicios financieros, número de sucursales bancarias por cada 100.000 habitantes adultos, es aun más baja, especialmente en África Subsahariana (excepto África Oriental).

En realidad, el número de cajeros automáticos y sucursales ha aumentado desde principios de la década de 2000, acercando los servicios a la población, pero los bancos también, sobre todo, aprovechando las nuevas tecnologías con aplicaciones móviles y banca online para atender mejor a la demanda. En cuanto a las operaciones de activo, los bancos continúan liderando la actividad de crédito a pesar de que una alta proporción de empresas no tiene acceso al crédito. Se $\triangleright$ 
CUADRO 4

PENETRACIÓN FINANCIERA EN ÁFRICA EN 2017

\begin{tabular}{|c|c|c|c|}
\hline & $\begin{array}{l}\text { Cuentas bancarias } \\
\text { por } 1.000 \text { adultos }\end{array}$ & $\begin{array}{l}\text { Sucursales bancarias } \\
\text { por } 100.000 \text { adultos }\end{array}$ & $\begin{array}{l}\text { Concentración de activos } \\
\text { de los } 5 \text { primeros bancos }\end{array}$ \\
\hline África & 476,00 & 8,75 & 80,49 \\
\hline África del Norte & 517,68 & 10,36 & 79,11 \\
\hline África Subsahariana & 465,58 & 8,34 & 80,84 \\
\hline África del Oeste & 402,14 & 6,81 & 77,99 \\
\hline 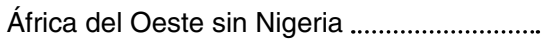 & 358,74 & 6,99 & 79,33 \\
\hline África Central & 230,53 & 6,89 & 84,26 \\
\hline África del Este & 754,30 & 13,06 & 73,80 \\
\hline África Austral & - & 6,62 & 87,31 \\
\hline 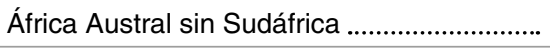 & 475,33 & 5,98 & 85,72 \\
\hline
\end{tabular}

estima que la brecha financiera de las pymes supera los 331.000 millones de dólares solo en África Subsahariana ${ }^{5}$. El problema principal es la falta de sistemas adecuados de información crediticia, que coloca a los bancos en una situación de riesgo muy elevado para desarrollar sus operaciones de activo. Como resultado, la tasa de morosidad de los bancos africanos se mantuvo en promedio en el $11,9 \%$ durante la última década, tres veces más alta que la media mundial ${ }^{6}$.

\subsection{Servicios financieros digitales y dinero móvil}

Con solo el $41 \%$ de los adultos bancarizados formalmente en África, la tecnología digital y móvil se ha convertido en la vía principal para mejorar el acceso a los servicios financieros del resto de la población, principalmente mujeres, jóvenes y población de las zonas rurales.

\footnotetext{
5 Datos anunciados durante el SME Finance Forum en 2018.
}

6 En el artículo siguiente se aporta más información sobre el desarrollo incipiente de los sistemas privados de información crediticia y el lanzamiento de la base de datos GEMS (Global Emerging Markets Risk Database) en 2019, que ha constituido un hito importante para el desarrollo de un sistema generalizado de información sobre riesgos financieros en África.
Según el informe GSMA de 20197, las cuentas de dinero móvil están disponibles en el $96 \%$ de los países, donde menos de un tercio de la población tiene una cuenta en una institución financiera formal.

De acuerdo con los datos de Global Findex (Demirgüç-Kunt et al., 2018), África Subsahariana lidera actualmente la adopción de dinero móvil, con casi el $21 \%$ de los adultos que poseen una cuenta de dinero móvil en 2017. Solo en 2019, el número de cuentas de dinero móvil registradas aumentó en 50 millones. Para aprovechar esta clientela potencial, los bancos están colaborando con operadores móviles y compañías fintech para introducir nuevos productos financieros ${ }^{8}$.

Las transferencias de efectivo es otro de los servicios financieros digitales más utilizados. En 2019 se registraron más de 690.000 millones de dólares en transacciones a nivel mundial (24\%), y África Subsahariana representó más del $60 \%$ de ese valor, con un aumento previsible durante la pandemia de 2020.

\footnotetext{
7 Global System for Mobile Communication o GSM Association. https://www.gsma.com/sotir/wp-content/uploads/2020/03/GSMA-State-ofthe-Industry-Report-on-Mobile-Money-2019-Full-Report.pdf

8 A título de ejemplo, en junio de 2020, la operadora de comunicaciones francesa Orange, en asociación con el grupo asegurador africano NSIA, anunció el lanzamiento del nuevo banco Orange Bank Africa en Costa de Marfil con la intención de extender su actividad a Senegal, Mali y Burkina Faso.
} 
El dinero móvil también está afectando positivamente la vida de los pequeños agricultores, que representan el $54 \%$ de la fuerza laboral en el África Subsahariana. El informe GSMA de 2019 muestra que alrededor del $10 \%$ de los proveedores de dinero móvil a nivel mundial colabora con empresas del sector agrícola. De este $10 \%$, el $75 \%$ está en África Subsahariana.

Aunque los servicios financieros digitales ya ayudan significativamente a hacer que las finanzas sean más accesibles, su potencial esta aún desaprovechado debido a la interoperabilidad limitada. Actualmente existen varios proyectos en marcha para abordar los problemas de interoperabilidad tanto a nivel nacional como regional. Los avances en este asunto serán la clave de la expansión de los sistemas de pago y un impulso decisivo para las transacciones comerciales privadas y las transferencias de remesas ${ }^{9}$.

\section{Financiación a largo plazo}

La disponibilidad limitada de financiación a largo plazo en los mercados africanos es una de las mayores preocupaciones debido a la enorme brecha existente para cubrir las necesidades de infraestructuras, vivienda de África y financiación empresarial, en particular de pymes. Actualmente, la financiación a largo plazo para infraestructuras en África proviene principalmente del extranjero, incluida la financiación pública.

El desarrollo de los mercados financieros africanos, aún relativamente pequeños y poco diversificados, tiene un papel destacado en la solución de este grave problema. Los cambios

9 También en este asunto se están haciendo progresos. Ghana introdujo un sistema de interoperabilidad de dinero móvil en 2018 que permite la transferencia de fondos entre redes de dinero móvil. La BCEAO (Banco Central de los Estados de África Occidental), en colaboración con el BAfD, está trabajando en la interoperabilidad de los sistemas de pago digital de los ocho países de África Occidental pertenecientes a la Unión Económica y Monetaria de África Occidental (UEMAO). experimentados en las últimas décadas han sido significativos para desarrollar una nueva gama de productos destinados a los inversores, tanto extranjeros como africanos.

\subsection{Mercados de capital y deuda}

Las bolsas de valores africanas siguen siendo pequeñas (en comparación con sus contrapartes de Asia o América Latina) y muy concentradas. La bolsa de valores de Johannesburgo (JSE) es la principal, y las cinco bolsas más grandes (Sudáfrica, Marruecos, Egipto, Nigeria y Kenia) representan aproximadamente el $90 \%$ de este total. $\mathrm{La}$ capitalización de las treinta bolsas de valores de África superó 1,5 billones de dólares en 2018. La capitalización de la JSE representaba 3,6 veces el PIB nominal del país en 2020, mientras que la proporción en el resto de los países africanos es mucho más baja (por ejemplo: Ghana, $8 \%$, Nigeria, $8 \%$, Egipto, $17 \%$ y Kenia, $24 \%$ ). Están muy lejos de las proporciones superiores al $100 \%$ normales en los países desarrollados, lo que refleja la ausencia de una masa crítica de empresas locales interesadas en cotizar. No obstante, el porcentaje de capitalización de mercado con respecto al PIB ha aumentado significativamente entre 2011 y 2019 , del $38 \%$ al $61 \%$ en promedio.

Debido a la fragmentación y el reducido volumen de transacciones, la liquidez sigue siendo un gran desafío en la mayoría de las bolsas africanas, a excepción de Sudáfrica y, en cierta medida, Egipto. Por lo general, la negociación se produce solo en un número limitado de valores, que representan la mayor parte de la capitalización de mercado, principalmente acciones financieras, de telecomunicaciones o inmobiliarias.

Esta situación ha empezado a cambiar gradualmente a lo largo de la última década. Desde finales de los noventa se introdujeron $D$ 
acuerdos de cotización cruzada que contribuyeron a un modesto repunte en los volúmenes de negociación. Posteriormente, el lanzamiento de los mercados regionales, principalmente en África del Oeste y Central ${ }^{10}$, ha contribuido a aumentar la liquidez. Este proceso de integración ha recibido un impulso definitivo con el proyecto de interconexión de las principales bolsas del continente (African Exchanges Linkage Project, AELP), liderado por la African Securities Exchanges Association (ASEA) con el apoyo del BAfD y la Cooperación Económica Corea-África ${ }^{11}$.

Desde la década de 2000, muchos países africanos también han desarrollado sus mercados de deuda como una fuente alternativa de financiación para sus economías. Los mercados de deuda pública son relativamente recientes y pocos países disponen de un mercado de deuda secundario (Sudáfrica, Nigeria, Ghana y Botsuana). La emisión total de bonos y letras ha pasado de 28.000 millones de USD en 2000 a más de 200.000 millones de USD anuales en promedio desde 2009, con un valor nocional de la deuda soberana en circulación por valor de aproximadamente 400.000 millones a finales de 2019. Los altos tipos de interés y el volumen elevado de emisiones soberanas han desplazado a los emisores corporativos ${ }^{12}$.

\footnotetext{
10 Bourse Régionale des Valeurs Mobilières (BRVM) y Bourse des Valeurs Mobilières de l'Afrique Centrale (BVMAC).

11 La ASEA fue creada en 1993 y hoy agrupa a veintisiete bolsas con el objetivo de promover el desarrollo de los mercados y alcanzar un volumen de capitalización medio superior al 123\% en 2023 (https:// african-exchanges.org/). El AELP es el proyecto más importante de la asociación que está también promoviendo acuerdo con otras bolsas fuera del continente (https://africanexchangeslink.com/). El interés suscitado por los bonos verdes y sociales es uno de los factores, junto con estos procesos de interconexión a nivel regional y mundial, que puede favorecer el despegue de los mercados financieros africanos.

12 PWC Africa publica anualmente, desde 2014, un informe de seguimiento de los mercados de capitales africanos que se ha convertido en la principal referencia (PwC's Africa Capital Markets Watch). La última edición disponible corresponde al año 2019 y es el quinto informe anual que cubre tanto los mercados de capital de valores como el de deuda, el sexto en lo que se refiere solo a los mercados de valores (https://www. pwc.com/ng/en/publications/africa-capital-markets-watch.html).
}

En general, los países africanos están logrando avances gracias a la introducción de nuevos productos más adaptados, las reformas de la regulación y la adopción de políticas económicas más adecuadas para atraer la inversión. A nivel nacional, el refuerzo de la capacidad del sector público está mejorando gradualmente la transparencia de los mercados. Las Instituciones Financieras de Desarrollo (IFD) multilaterales y bilaterales están apoyando la capacitación de los centros encargados de la gestión de la deuda pública para mejorar la transparencia, extender la curva de rendimientos de manera creíble y aumentar la atracción de inversores institucionales locales e internacionales. Entre las actividades destaca la Iniciativa de Mercados Financieros Africanos (AFMI, por sus siglas en inglés) ${ }^{13}$.

Otra de las características principales de los mercados financieros en África, que está también recibiendo atención en los últimos años, es la escasez de oferta de financiación en moneda local a largo plazo, más allá de cinco años, en la mayoría de los mercados. Este tipo de financiación es esencial para la inversión en los sectores prioritarios de infraestructura y vivienda. En 2017, varias instituciones, agrupadas en torno a la iniciativa MFW4A, lanzaron la Africa Long-Term Finance Initiative (ALTFI) ${ }^{14}$.

13 Promovida por el BAfD, la iniciativa African Financial Markets Initiative contribuye a mejorar la información fiable y actualizada a través de una red de funcionarios de enlace (liason officers) en treinta bancos centrales africanos. Los dos principales elementos son el African Domestic Bond Fund (ADBF), primer ETF (Exchange Traded Fund de deuda en Africa), y la African Financial Markets Database (AFMD).

En 2017 se realizó también la primera emisión de deuda vendida exclusivamente a través de banca telefónica en Kenia (M-Akiba, https:// www.m-akiba.go.ke/).

14 El principal objetivo de la ALFTI (https://www.mfw4a.org/our-work/ africa-long-term-finance-initiative-altfi) es la transparencia y la información disponible en este mercado. Para ello se ha desarrollado una base de datos con un mecanismo de evaluación (scoreboard) para poner a disposición de los principales inversores, donantes y Gobiernos la información de calidad sobre los instrumentos disponibles, operaciones y demanda. La iniciativa realiza también diagnósticos a nivel nacional y regional. Los tres socios principales de la ALFTI son el BAfD, el Ministerio Federal Alemán de Cooperación Económica y Desarrollo, BMZ/GIZ y FSDA, Financial Sector Deepening Trust for Africa, financiado por la cooperación británica. 


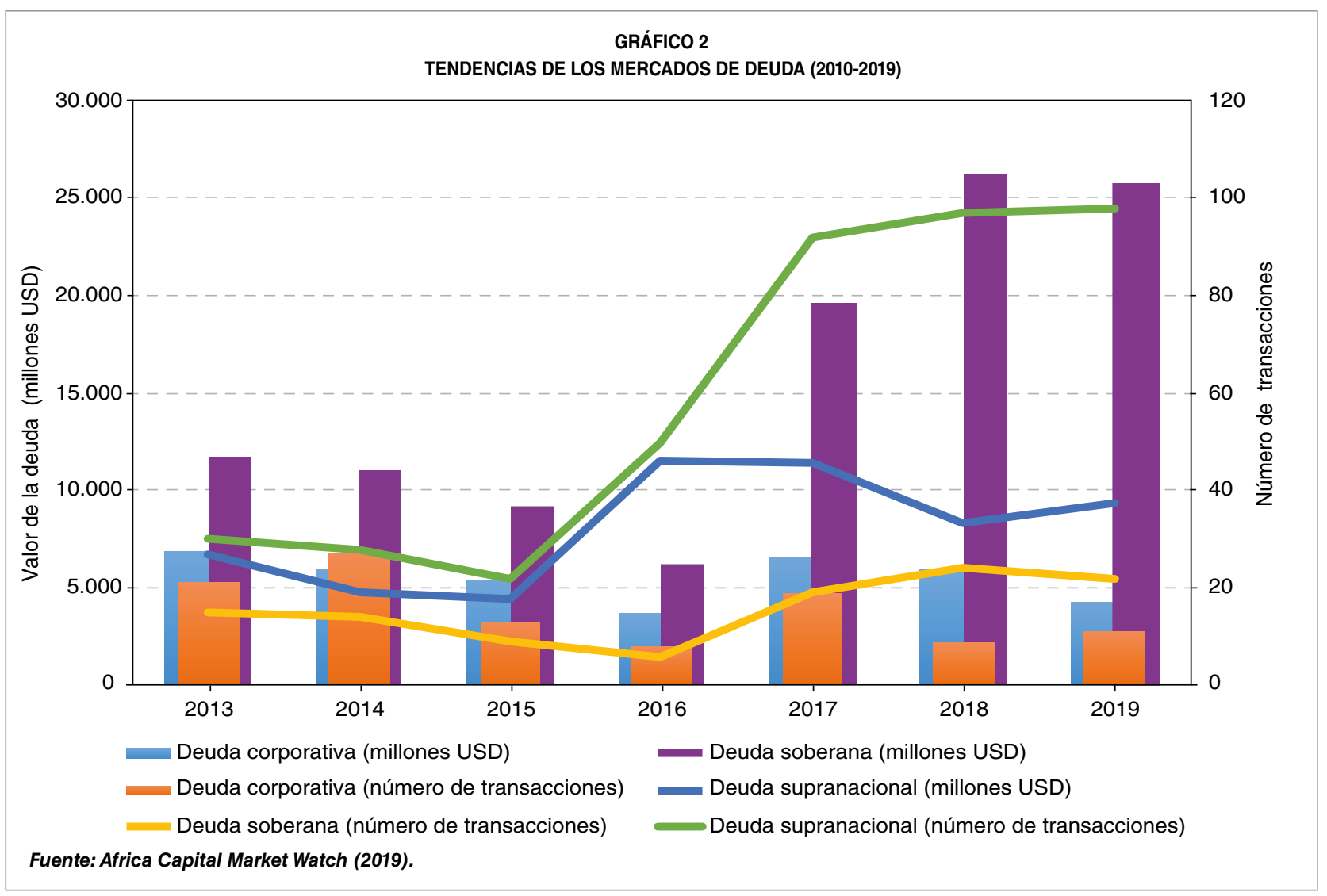

\subsection{Inversores institucionales}

Dado el reducido espacio fiscal y la disminución de la AOD (Asistencia Oficial para el Desarrollo) ${ }^{15}$, existe un consenso generalizado sobre el nuevo enfoque para obtener la financiación necesaria para el cumplimiento de los ODS en África. Este pasa por el desarrollo del sistema financiero con una atención particular a los inversores institucionales, tanto africanos como internacionales, que están llamados a desempeñar un papel fundamental en el aumento del acceso a la financiación privada a largo plazo.

La implicación de los inversores institucionales, fondos de pensiones, fondos soberanos

15 La financiación de los Objetivos de Desarrollo Sostenible de Naciones Unidas en África requiere entre 600.000 y 700.000 millones de dólares al año de acuerdo con las estimaciones realizadas por Naciones Unidas (UNCTAD, 2016).
(SWF) y compañías de seguros facilitaría la movilización de los recursos necesarios para ampliar la financiación de proyectos de infraestructura y servicios sociales básicos.

El sector de seguros en África está aún en una fase incipiente de desarrollo, pero con un potencial de crecimiento mayor en comparación con otras regiones. La tasa de penetración se sitúa en torno al 2,8\%, en comparación con el $9 \%$ de los países de la OCDE y menos de la mitad del promedio mundial estimado en $6,3 \%$. Existen grandes disparidades regionales en todo el continente, y Sudáfrica tiene una tasa de penetración de mercado del $14 \%$. La industria de seguros está valorada en aproximadamente 68.000 millones de USD en términos de primas emitidas brutas en 2018.

Varios países han emprendido también reformas estructurales para crear un entorno propicio para nuevos planes de pensiones $D$ 
privados más dinámicos. Los cambios han contribuido a un crecimiento en los activos de los fondos de pensiones, por ejemplo, en Nigeria, donde los activos administrados por estos fondos han pasado de 7.000 millones de dólares en diciembre de 2008 a 30.000 millones en diciembre de 2020. Los activos de los fondos de pensiones en toda África se estiman en 372.000 millones de dólares en 2018 (293.000 millones en 2008). El crecimiento se ha atribuido en gran medida al desarrollo económico, el ascenso de la clase media, la mejora del acceso a los mercados financieros, los cambios regulatorios y los avances tecnológicos que están atrayendo a más personas a los sistemas de previsión social. A pesar de las reformas regulatorias de los sistemas de pensiones, la reticencia de los gestores de estos fondos junto con las limitadas oportunidades de diversificación del riesgo han sido las barreras principales que han limitado la movilización de estos recursos para la inversión productiva a más largo plazo.

Durante la última década se han desarrollado nuevos instrumentos financieros, fondos especializados de infraestructuras ${ }^{16}$, vehículos financieros estructurados que combinan diversas fuentes de financiación privada y pública con algún tipo de garantía, para atraer precisamente los fondos más conservadores. Todos estos aspectos se desarrollarán con más detalle en los próximos artículos.

Sería tan solo necesario mencionar, para concluir esta sección, que la crisis financiera de 2008 y el debate sobre los ODS han puesto de nuevo sobre la mesa el papel del Estado en

\footnotetext{
16 Numerosos fondos privados de infraestructuras han surgido recientemente y se agrupan en torno a la African Private Equity and Venture Capital Association (AVCA, https://www.avca-africa.org/).

En este área de la financiación de infraestructuras destaca un nuevo instrumento público especializado, el Fondo Africa50 (https://www. africa50.com/), creado en 2015 por el BAfD y otros veintisiete Gobiernos africanos y dos bancos centrales.
}

la economía, veinte años después de la liberalización de los años noventa. Como consecuencia, se ha renovado interés por las IFD, que también se analizará con mayor detalle en los siguientes artículos ${ }^{17}$.

\section{Regulación financiera y supervisión}

La crisis financiera mundial de 2007/2008 puso en primer plano numerosas fallas en los mecanismos de regulación y supervisión e hizo que se prestase más atención a la correlación entre el desarrollo del sector financiero y la estabilidad financiera y los vínculos entre los sistemas financieros y la economía real.

\subsection{Alineamiento con los estándares globales}

Para garantizar el buen funcionamiento de los sistemas financieros, los Gobiernos africanos se comprometieron a alinear la regulación y supervisión financieras con las mejores prácticas internacionales. Como resultado, los sistemas financieros son más estables, con sectores bancarios mejor capitalizados e incluso con exceso de liquidez.

Los estándares prudenciales globales representan un esfuerzo importante para reducir la probabilidad de otra crisis financiera sistémica. En consecuencia, los responsables $D$

\footnotetext{
17 Existen en África alrededor de 140 IFD y bancos nacionales y regionales de desarrollo, de las que 67 instituciones nacionales son miembros de la Asociación de Instituciones de Financiamiento para el Desarrollo en África (Association of African Development Finance Institutions, AADFI). Esta reúne también a otras doce organizaciones a escala regional y continental y apoya la capacitación y la autoevaluación de sus miembros en el marco de sus «Prudential Standards, Guidelines and Rating System for African Development Banks and Finance Institutions" (PSGRS). Este interés por las IFD ha recibido un nuevo impulso en la Cumbre Finance in Common de 2020, donde se lanzó una nueva base de datos especializada en los Bancos Públicos de Desarrollo (PBD, https://afdshiny.shinyapps.io/developmentbanksdatabase/).
} 
políticos africanos enfrentan una mayor presión para acelerar la adopción de regulaciones internacionales, en particular las recomendaciones de Basilea II, Basilea III, las normas internacionales de información financiera (NIIF) y el Grupo de Acción Financiera Internacional (GAFI).

En este contexto, el continente presenta diferentes niveles en cuanto a la aplicación de las recomendaciones. Algunos países africanos han optado por avanzar hacia la plena aplicación del nuevo marco de Basilea III, mientras que otros prefieren un enfoque gradual y personalizado de Basilea II y III. Entre los segundos, las autoridades nigerianas decidieron implementar solo los aspectos que se determinaron como adecuados para su país. Por el contrario, Sudáfrica y la región de la zona CFA, bajo la supervisión de la BCEAO (Banque Centrale des États de l'Afrique de l'Ouest), se han comprometido con las normas en su totalidad.

Al igual que los bancos centrales, el reconocimiento unánime de la importancia de la gobernanza como un ingrediente clave para el desarrollo del sector financiero ha obligado a los reguladores de todos los sectores financieros en África, seguros, pensiones y mercado de valores a introducir los principios de buen gobierno corporativo en las áreas bajo su responsabilidad.

\subsection{Mejora de la regulación y refuerzo de las entidades de supervisión financiera}

Tradicionalmente, los riesgos de crédito, liquidez y mercado son las principales preocupaciones de los reguladores y supervisores. Sin embargo, el ritmo acelerado de adopción de las nuevas tecnologías por las fintech y la diversificación de los servicios financieros ha ejercido una mayor presión sobre los reguladores y supervisores para desarrollar y aplicar una regulación mejor adaptada.

Los nuevos modelos de negocio, a menudo, traspasan las fronteras de los servicios financieros tradicionales y requieren una fuerte cooperación entre reguladores y supervisores de los sectores financiero y de telecomunicaciones. Al mismo tiempo que se aprovechan los beneficios de las nuevas tecnologías financieras, es necesario garantizar que los riesgos que plantean dichas innovaciones para la estabilidad financiera se gestionen y mitiguen adecuadamente para garantizar que los sistemas financieros desempeñen plenamente su papel de apoyo al crecimiento económico.

En este nuevo contexto, una de las principales limitaciones observadas es que los reguladores y supervisores carecen de la capacidad y los recursos para mantenerse al día con la evolución del mercado y supervisar la creciente complejidad del sector financiero. Las amenazas nuevas y emergentes, incluidos el blanqueo de dinero, el fraude y los ataques cibernéticos, exigirán una mayor inversión en sistemas y capacidades del personal. No es un problema exclusivo de África, pero afecta de manera particular a las autoridades en el continente debido a la menor capacidad y una más rápida adopción de las nuevas tecnologías por la población.

La experiencia reciente, durante la crisis financiera mundial de 2008, ha confirmado la importancia de introducir un sistema de regulación financiera basado en el análisis del riesgo potencial para equilibrar los objetivos de estabilidad, integridad, inclusión y protección del consumidor. La actual crisis de la pandemia de COVID-19 ha revelado la vulnerabilidad creciente de los sistemas financieros africanos a los choques endógenos y exógenos. 
Por último, las autoridades reguladoras y supervisoras de África están integrando cada vez más los riesgos derivados del cambio climático en el seguimiento de la estabilidad financiera y la supervisión microprudencial. El cambio climático como fuente de riesgo financiero puede afectar los balances y los modelos de negocio de los bancos comerciales de muy diferentes formas. El propósito es orientar al sector bancario hacia la transición a una economía de bajas emisiones de carbono aplicando una regulación adaptada y una supervisión adecuada.

Consciente de la necesidad de contribuir a los esfuerzos en curso para fortalecer los marcos regulatorios y de supervisión bancarios en el continente, la Comunidad de Supervisores Bancarios Africanos (Community of African Banking Supervisors, CABS), establecida por la Asociación de Bancos Centrales Africanos (Association of African Central Banks, AACB) con el apoyo de MFW4A, se está convirtiendo en el principal instrumento de colaboración de los reguladores africanos para la supervisión transfronteriza y el cumplimiento de las normas internacionales, así como el desarrollo de las reformas prioritarias y el dialogo con otros supervisores a nivel internacional.

\section{Conclusión}

El peso del sector financiero en África es aún muy bajo tanto en términos absolutos, comparado con el resto del mundo, como en términos relativos con respecto al PIB de cada país. Sin embargo, en las secciones anteriores se han esbozado los principales avances que se han producido en las dos últimas décadas y que están poniendo las bases para un desarrollo sostenido e inclusivo de los servicios financieros que contribuya al desarrollo económico general.
Se debe destacar, a modo de conclusión, que la mayoría de los aspectos señalados están favoreciendo una mayor integración regional tanto a través de la expansión de los grupos financieros privados y la interconexión de los mercados como de la colaboración pública para la armonización y mejora de la regulación y la supervisión a escala continental.

Esta integración, junto con el avance significativo de la innovación financiera y la adopción de las tecnologías de comunicación, es el principal factor que contribuirá a resolver la fragmentación del que previsiblemente será uno de los principales mercados mundiales a partir de 2050 en el continente más poblado.

El desarrollo de los grupos financieros panafricanos es otro aspecto importante que requiere atención por las implicaciones potenciales en las relaciones económicas dentro de África y de ésta con el resto del mundo.

La colaboración entre MFW4A y AMENET en la elaboración de este trabajo es tan solo un primer paso en el inicio de una relación que se espera que sea fructífera en los próximos años. Esta colaboración servirá para involucrar a más investigadores africanos y europeos, lo cual redundará en la mejora de la capacidad de análisis y en el enriquecimiento del debate de las políticas económicas y sociales sostenibles.

\section{Bibliografía}

Banco Africano de Desarrollo (2020). Developing Africa's Workforce for the Future. African Economic Outlook 2020. https://www.afdb.org/en/documents/african-economic-outlook-2020

Banco Europeo de Inversiones (2020). Banking in Africa: financing transformation amid uncertainty. https://op.europa.eu/en/publication-detail//publication/7b3c1887-5f5b-11ea-b735-01 aa75ed71a1 
Boumahdi, I. (2019). Le positionnement du secteur bancaire marocain en Afrique: réalité et perspectives de renforcement. DEPF Policy Africa. Ministère de l'Economie et des Finances du Royaume de Maroc. https://www.finances.gov.ma/ Publication/depf/2019/Policy\%20Africa_secteur\%20bancaire.pdf

Chironga, M., Cunha, L., De Grandis, H., y Kuyoro, M. (2018). Roaring to Life: Growth and Innovation in African Retail Banking. Global Banking Practice. McKinsey and Company. https://www. mckinsey.com/ /media/mckinsey/industries/financial\%20services/our\%20insights/african $\% 20$ retail $\% 20$ bankings $\% 20$ next $\% 20$ growth\%20frontier/roaring-to-life-growth-and-innovation-in-african-retail-banking-web-final.ashx

Demirgüç-Kunt, A., Klapper, L., Singer, D., Ansar, S., y Hess, J. (2018). The Global Findex Database 2017. Measuring financial inclusion and the fintech revolution. World Bank Group. https://openknowledge.worldbank.org/handle/10986/29510

Mlambo, K., Kasekende, L., y Murinde, V. (2012). Comparative overview of bank regulatory experiences and the impact of bank competition and intermediation efficiency in Africa. In V. Murinde (Ed.), Bank Regulatory Reforms in Africa. Palgrave Macmillan Studies in Banking and Financial Institutions.
ONU/DESA (2017). World Population Prospects: The 2017 Revision (Working Paper No. ESA/P/ WP/248). United Nations Department of Economic and Social Affairs, Population Division. Nueva York.

Raga, S., y Tyson, J. (2021a). Impact of pan-African banks on financial development in sub-Saharan Africa. Synthesis report. Development and Economic Growth Research Programme (DEGRP). https://degrp.odi.org/wp-content/uploads/ 2021/03/ODI-DEGRP-ImpactPanAfricanBanksSynthesis-Report-March21-FINAL040321.pdf

Raga, S., y Tyson, J. (2021b). Sub-Saharan Africa's interbank markets: progress, barriers and policy implications. Synthesis report. Development and Economic Growth Research Programme (DEGRP). https://degrp.odi.org/wp-content/uploads/2021/02/ Sub-Saharan-Africas-interbank-markets-DEGRP2021-Synthesis-Report.pdf

The Economist (26 de marzo de 2020). Africa is changing so rapidly, it is becoming hard to ignore. Special report. https://www.economist.com/ special-report/2020/03/26/africa-is-changingso-rapidly-it-is-becoming-hard-to-ignore

UNCTAD (2016). World Investment Report 2016: Investor Nationality: Policy Challenges. United Nations. https://unctad.org/system/files/officialdocument/wir2016_en.pdf 
